\title{
An Automated Rotor Time Constant Measurement System for Indirect Field-Oriented Drives
}

\author{
CHU WANG, DONALD W. NOVOTNY, FELlow, IEEE, AND THOMAS A. LIPO, FELLOW, IEEE
}

\begin{abstract}
A new automated scheme to measure the open-circuit rotor time constant and set the slip gain of a field-oriented controller is presented. The test utilizes the pulsewidth-modulated inverter of the drive itself and is conducted at zero speed so that the result can be used for automated calibration of the slip gain in the controller of an indirect field-oriented drive system. The accuracy of the test is verified by a simple measurement that checks for the existence of a linear relation between the input torque command and output torque at steady state.
\end{abstract}

\section{INTRODUCTION}

F ELD-ORIENTATION systems that employ an encoder or resolver typically utilize a slip calculator to establish the rotor flux angle with respect to the stator MMF. In such systems accurate knowledge of the machine rotor time constant is essential. If the time constant used in setting the slip controller gain is incorrect, machine performance is degraded for both transient and steady-state conditions [1], [2]. Correct "tuning" of the slip controller is an expensive and timeconsuming process typically requiring knowledge of the motor parameters from the locked rotor and no-load test. While such an expense can be justified on a special-purpose servo drive, it becomes impractical when applied to situations where low cost is imperative or where the inverter and the motor arrive at the installation from different manufacturers. Such applications, however, comprise the vast majority of motor drive installations in the United States. For field-oriented control to be practical on a large-scale basis it is important, if not crucial, that a low-cost scheme be developed to set the controller gains automatically without the need for the extensive testing now required.

A considerable body of literature exists concerning measurement or adjustment of the estimated rotor time constant during normal operation [3]-[6]. However, none of these methods have been concerned with determination of the time constant prior to startup of the drive. This type of measurement is essential to the development of a system that is "selftuning" in the sense of automatically determining the proper slip calculation gain prior to commissioning of the drive with

Paper IPCSD 87-16, approved by the Industrial Drives Committee of the IEEE Industry Applications Society for presentation at the 1986 Industry Applications Society Annual Meeting, Denver, CO, September 28-October 3. This work supported by the Wisconsin Electric Machines and Power Electronics Consortium (WEMPEC).

C.-G. Wang is with Gould Inc., 2701 North Green Bay Road, Racine, WI 53404

D. W. Novotny and T. A. Lipo are with the Department of Electrical and Computer Engineering, University of Wisconsin-Madison, 1425 Johnson Drive, Madison, WI 53706-1691.

IEEE Log Number 8716202. an unknown motor. The development of such a self-tuning field-oriented controller that automatically determines the proper slip calculator gain at startup is the subject of this paper.

The basic measurement concept set forth in this paper is to utilize the same current-regulated inverter that normally drives the motor to perform a sequence of tests prior to actual starting of the drive. To achieve full automation of the test and slip gain adjustment procedure, a system that requires no external constraints on the motor or controller is necessary (i.e., no mechanical means of blocking the rotor, for example). It is also generally desirable to utilize a null-seeking controller, as opposed to a direct measurement, in systems of this type. The system that is described in this paper meets these goals by employing single-phase excitation to achieve locked rotor tests without need for physically locking the rotor. The test is carried out as a sequence of single-phase measurements with varying excitation frequency until a null is reached in the detection system. At the null point the proper slip frequency for field orientation is known and the slip calculator gain can be set to the correct value. The drive is then ready for normal operation.

\section{Automated Tuning System}

Fig. 1 is a block diagram of the automatic tuning system proposed in this paper. In the experimental system to be described, the block labeled "computer" was an IBM PC-AT, which was used to control the testing process. In a practical field-oriented controller, this function could be combined into the system controller, which carries out the normal fieldorientation requirements. The feedback signal from one of the motor line-to-line voltages is the only additional signal required to implement the automated tuning process. The remainder of the self-tuning system is entirely software-based and utilizes the normal command channels of the field-oriented controller. The test simply requires a series of programmed current commands to be delivered to the inverter. The information received from the voltage feedback loop is then processed to determined the rotor time constant.

Principle of the Tuning Algorithm

As is well known [7]-[9], field-oriented control concerns a method of manipulating the stator current and slip frequency such that the rotor flux and torque are independently controlled. This concept is illustrated in Fig. 2 for steady-state conditions. The circuit in Fig. 2(a) is the conventional steadystate equivalent circuit of an induction motor. In Fig. 2(b) this 


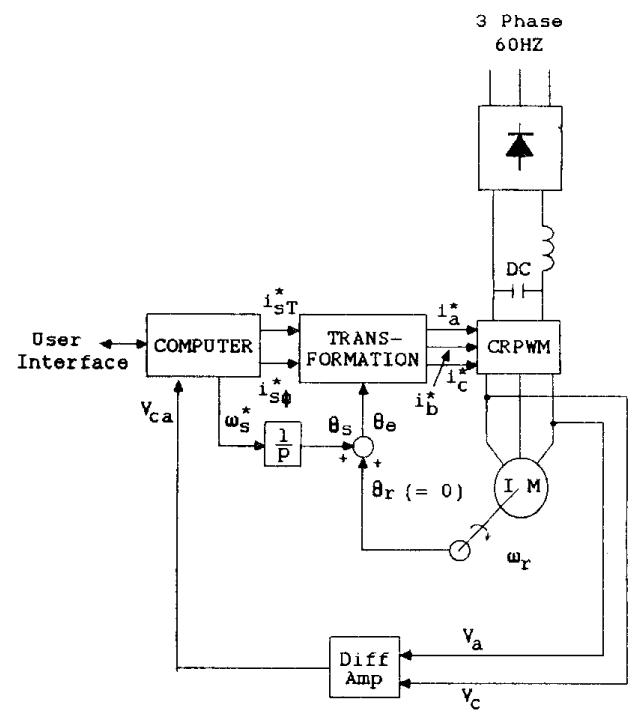

Fig. 1. Automated tuning system for rotor time constant measurement.
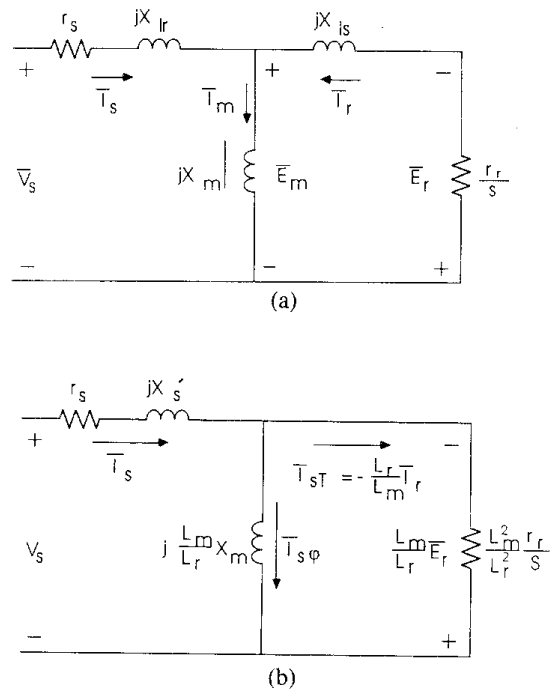

Fig. 2. (a) Conventional induction motor equivalent circuit. (b) Modified equivalent circuit.

conventional circuit is modified by choosing a different referral ratio for the rotor current such that the rotor leakage reactance is eliminated. The two components of the stator current in Fig. 2(b), $I_{s \phi}$ and $I_{s T}$, become the rotor flux- and torque-producing components [10]. This fact is easily demonstrated since $I_{s \phi}$ clearly produces the rotor voltage $E_{r}$, which is proportional to the rotor flux. That is, in the steady state,

$$
I_{s \phi} X_{m}=E_{r}=\omega_{e} \lambda_{r}
$$

where $I_{s \phi}$ is the amplitude of the current in the magnetizing branch of Fig. 2(b), from which

$$
\lambda_{r}=L_{m} I_{s \phi} .
$$

The electromagnetic torque can be written in terms of $E_{r}$ and $I_{s T}$; the steady-state voltage and current amplitude of the rotor branch of Fig. 2(b) as

$$
\begin{aligned}
T_{e} & =3 \frac{P}{2} \frac{L_{m}}{L_{r}} \frac{E_{r} i_{s T}}{\omega_{e}} \\
& =3 \frac{P}{2} \frac{L_{m}}{L_{r}} \lambda_{r} I_{s T}
\end{aligned}
$$

which demonstrates the role of $I_{s T}$ in controlling the torque The slip relation, which is the basis of indirect field orientation, results from equating the voltages produced by $I_{s \oplus}$ and $I_{s T}$, which yields

$$
\frac{L_{m}^{2}}{L_{r}} \omega_{e} I_{S \phi}=\frac{L_{m}^{2} r_{r}}{L_{r}^{2}} \frac{1}{S} I_{s T}
$$

or

$$
S \omega_{e}=\frac{r_{r}}{L_{r}} \frac{I_{s} T}{I_{S \phi}}
$$

The results expressed in (2)-(6) are valid for all operating conditions. At zero speed the circuit of Fig. 2(b) is also valid for transient conditions since all speed voltages are zero and the induction motor is effectively reduced to that of a transformer. Furthermore, the circuit can be viewed as representing single-phase operation at zero speed since the coupling between phases of a two-phase $(d-q)$ model vanishes at zero speed.

If a single-phase sinusoidal test current $i_{s}=I_{s} \cos \omega_{e} t$ is supplied to the machine and sufficient time is allowed to reach steady state, the current will divide according to (6) with $S=$ 1.0:

$$
\frac{I_{s T}}{I_{s \phi}}=\frac{\omega_{e} L_{r}}{r_{r}}
$$

where $I_{s T}$ and $I_{s \phi}$ are the in-phase and quadrature components of $I_{s}$ with respect to the voltage $E_{r}$. The test procedure consists of choosing values for $I_{s \phi}$ and $I_{S T}$ and applying the sinusoidal test current of amplitude

$$
I_{s}=\sqrt{I_{s T}^{2}+I_{s \downarrow}^{2}}
$$

at an arbitrary frequency $\omega_{e}$. After sufficient time for steadystate conditions to be attained $\left(t \gg L_{r} / r_{r}\right)$, the test current is switched to dc at the level $I_{s \phi}$ as shown in Fig. 3. Neglecting the very small initial effect of the stator leakage inductance, the stator voltage that will be observed upon switching to a dc level is the sum of the time derivative of the air gap flux and the stator IR drop. This transient component of voltage can be expressed as

$$
\nu_{S t}=-\frac{1}{\tau_{r}}\left[\lambda_{r}(T)-L_{m} I_{s \phi}\right] e^{-(t-T) / \tau_{r}}+I_{s \phi} r_{s}
$$

where $T$ is the instant of switching to dc and $I_{s \phi}$ is the stator current at the instant of switching.

If the command slip frequency is larger than the value for 


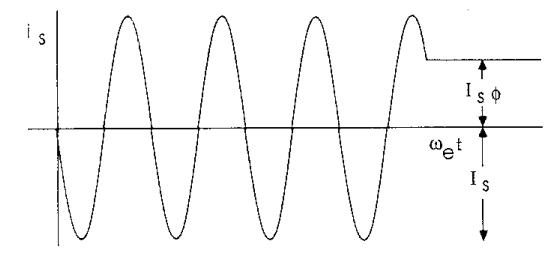

Fig. 3. Stator current test signal for rotor time constant measurement.

correct tuning, then the actual magnetizing current, and thus the rotor flux, are below the command values. After the command current switches to a dc value (which is the same as the commanded flux-producing component of current), the instantaneous current in the magnetizing branch will be smaller than the instantaneous stator current. Some current will be trapped in the branch containing the rotor resistor since the current that flows through an inductor cannot be changed instantaneously. The directions of the currents are shown in Fig. 4(a). The transient voltage across the magnetizing branch is determined by the instantaneous current in the rotor resistor branch, and the stator terminal voltage is the sum of the transient voltage plus the stator IR drop. The stator leakage drop can be be ignored because it is insignificant compared to other quantities. Eventually, of course, all the rotor current will flow through the magnetizing branch because the magnetizing branch is a short circuit at zero frequency, and in the steady state the rotor flux will again equal the commanded value.

On the other hand, if the command slip frequency is too small, then the magnetizing current and the rotor flux are too large before the current switching. The directions of the instantaneous currents after switching the current to a dc level are shown in Fig. 4(b). Notice that the direction of the current in the rotor resistor branch is reversed from the last case because the instantaneous magnetizing current is larger than the stator current. The trapped rotor current will eventually again decay to zero as the rotor flux decays to the command value. The stator terminal voltage will now be initially smaller than the steady-state voltage because the polarity of the transient voltage across the magnetizing inductance is opposed to the stator IR drop.

It is clear that the trapped current will equal zero only when the command slip frequency is the same as the correctly tuned value. In this case the magnetizing current will equal the commanded flux, producing a component of current both before and after the current switching such that there will be no voltage induced across the magnetizing branch. The stator terminal voltage will simply equal the stator IR drop. The slip calculator is correctly tuned under this condition.

\section{Test Procedure}

From the description above, a test procedure for the rotor time constant can readily be established. Specifically, by use of the current-regulated pulsewidth-modulated inverter, a single-phase sinusoidal current can be supplied to two phases of the induction machine with the current in the third phase controlled to zero. The rotor will not rotate since the machine is operating as a single-phase machine and need not be

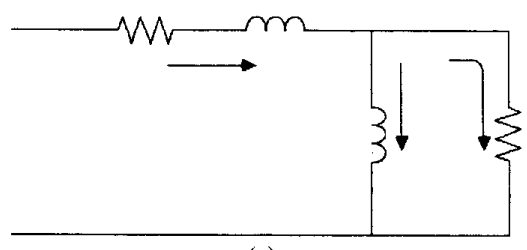

(a)

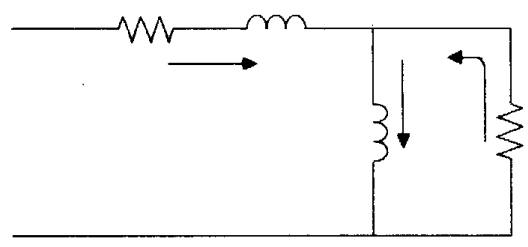

(b)

Fig. 4. Directions of motor currents during rotor time constant test. (a) Command frequency too large. (b) Command frequency too small.

locked. After the flux reaches the steady state, the stator current is switched to a dc current and the point of switching is fixed at the point where the stator current instantaneously equals the commanded magnetizing current $I_{s \phi}$. The value of the magnetizing current is found from a no-load test and depends on the desired flux level. It should be noted that the applied inverter frequency is the same as the slip frequency since the rotor speed is zero. The inverter frequency is adjusted until the voltage transient is minimum. The rotor time constant can then be calculated as

$$
\tau_{r}=\frac{1}{\omega_{s}} \frac{I_{s T}^{*}}{I_{s \phi}^{*}}=\frac{1}{\omega_{s}} \frac{\sqrt{I_{s}^{2}-I_{s \phi}^{2 *}}}{I_{s \phi}^{*}}
$$

where $\omega_{s}$ is the slip frequency.

\section{Transient Flux Linkage Error Characteristic}

Using the circuit of Fig. 2(b) the behavior of the rotor flux before and after the current switching can be calculated. The flux error for a certain 3-hp machine (see Appendix) is plotted against the slip frequency (inverter frequency) in Fig. 5. The horizontal scale has been normalized with respect to the correct slip frequency so that the error becomes zero when the actual slip frequency matches the correct slip frequency $(1.0$ on Fig. 5) and also when the slip frequency equals zero. The curve to the right of the correct slip frequency represents an underflux situation, i.e, the actual rotor flux is below the desired flux level. The further the slip frequency is from the correct value, the smaller the value of the flux and the greater will be the voltage transient. On the other hand, when the slip frequency is too small, the flux error first increases and then decreases as the slip frequency decreases. The error will finally again reach zero when the slip frequency reaches zero. This behavior should be expected since field orientation is always attained in the case of $\mathrm{dc}$.

\section{Choice of Test Currents}

The test procedure outlined in the last section involves the choice of the two command currents, $I_{s T}^{*}$ and $I_{s \phi}^{*}$. Normally a field-oriented drive system is operated at a fixed flux condition 


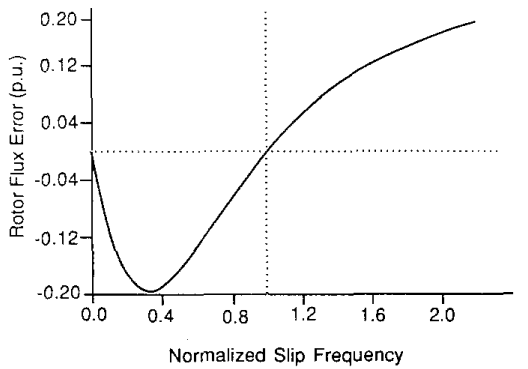

Fig. 5. Calculated flux error versus commanded slip frequency for 3-hp machine using proposed time constant test.

to obtain fast torque response. In other words, the magnetizing current must be held constant to provide the desired flux in the machine when the command slip frequency is correct. The value of the magnetizing inductance, as well as the rotor time constant, depends upon the value of the magnetizing current. It is desirable to perform the test at a fixed magnetizing current level which will produce the desired flux in the machine. Theoretically, the greater the ratio of the currents, the better will be the resultant accuracy. However, the choice of $I_{s T}^{*}$, or aiternatively $I_{s T}^{*} / I_{s \phi}^{*}$, should be selected with due regard to how much flux error can be tolerated when the time constant is incorrect.

In Fig. 6 the flux error is plotted against the current ratio $I_{s T}^{*} / I_{s}^{*}$. Each curve represents a particular setting of the gain in the controller. The top curve has an estimated time constant (gain) that is one-tenth the correct value of $\tau_{r}^{*}=0.1 \tau_{r}$. The bottom curve has a gain of $\tau_{r}^{*}=5 \tau_{r}$. It can be noted that the curve with the smaller estimated value levels out faster than the curve with a larger value. This phenomenon can be explained by the phasor diagram in Fig. 7, which is constructed for a value of $\tau_{r}^{*}=0.1 \tau_{r}$. The first diagram corresponds to a current ratio $\left(I_{s T}^{*} / I_{s \phi}^{*}\right)$ of 0.5 , the second diagram a ratio of 1 , and the last diagram a ratio 2 . It is apparent that the magnitude of the rotor flux does not change significantly from the second diagram to the third because the position of the rotor current is nearly the same. The rotor current position angle can be calculated by

$$
\theta=\tan ^{-1} \omega_{s}^{*} \tau_{r}=\tan ^{-1} \frac{\tau_{r}}{\tau_{r}^{*}} \frac{I_{s T}^{*}}{I_{s \phi}^{*}}
$$

where $\omega_{s}^{*}$ is the command slip frequency. The rate of change of the angle variation slows down when the angle approaches $90^{\circ}$. The smaller the ratio of $\tau_{r}^{*} / \tau_{r}$, the faster the angle reaches $90^{\circ}$. In practice the curve will level out even faster because of saturation. The curves suggest that a very large current ratio is not necessary in measuring the rotor time constant. This conclusion is important since with large current ratios the heating of the machine would pose a major problem. In the experimental study a current ratio of $2: 3$ has been employed.

\section{APPROACH TO FILTERING}

Because of the pulsewidth-modulated (PWM) inverter switching properties, filters will clearly be required to measure the voltage transients. In a hysteresis currentregulated PWM (CRPWM) system, unwanted harmonic

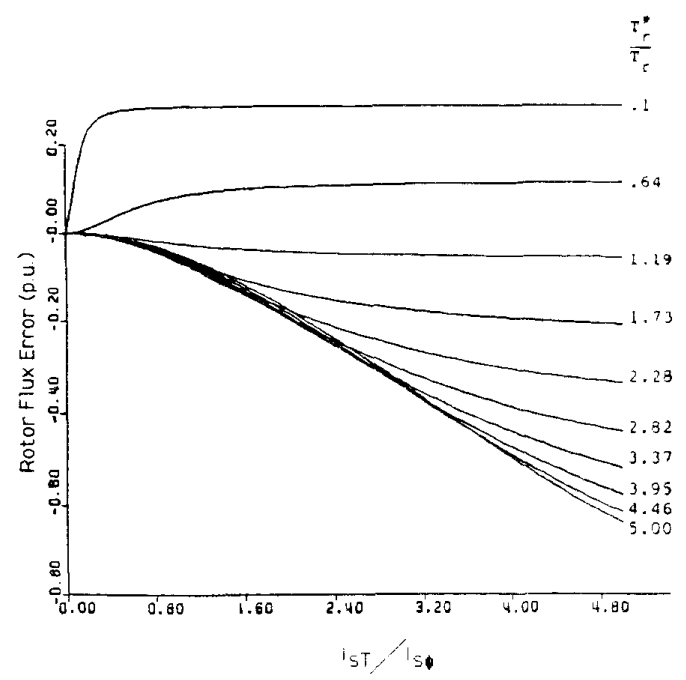

Fig. 6. Calculated flux transient for different input current ratios
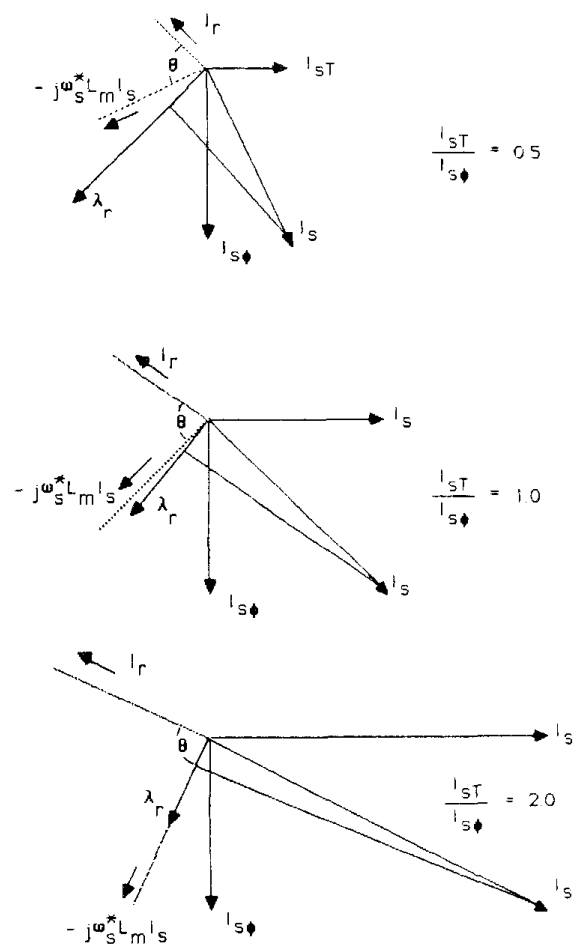

Fig. 7. Phasor diagrams showing rotor flux linkage for different current ratios with $\tau_{r}^{*}=0.1 \tau_{r}$

components will be scattered over the entire frequency domain. In a sine-triangle modulation type CRPWM, however, the harmonic components are fixed in bands about the harmonic frequencies of the triangle signal [11]. A typical voltage waveform of a sine-triangle-type CRPWM is shown in Fig. 8, and the corresponding power spectrum is shown in Fig. 9. The fundamental frequency of the voltage is $35 \mathrm{~Hz}$, and the frequency of the triangular signal is $1800 \mathrm{~Hz}$. It is clear that the major harmonic components are centered around the 


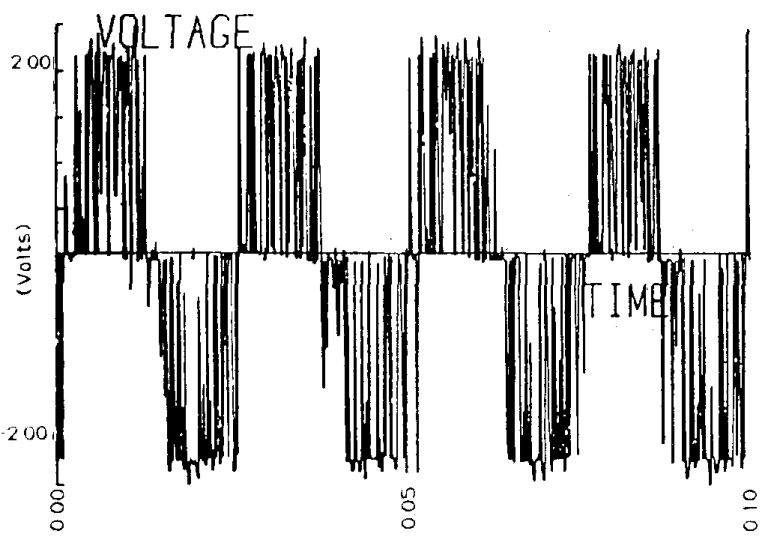

Time (Seconas)

Fig. 8. Typical voltage waveform with sine-triangle-type CRPWM inverter.

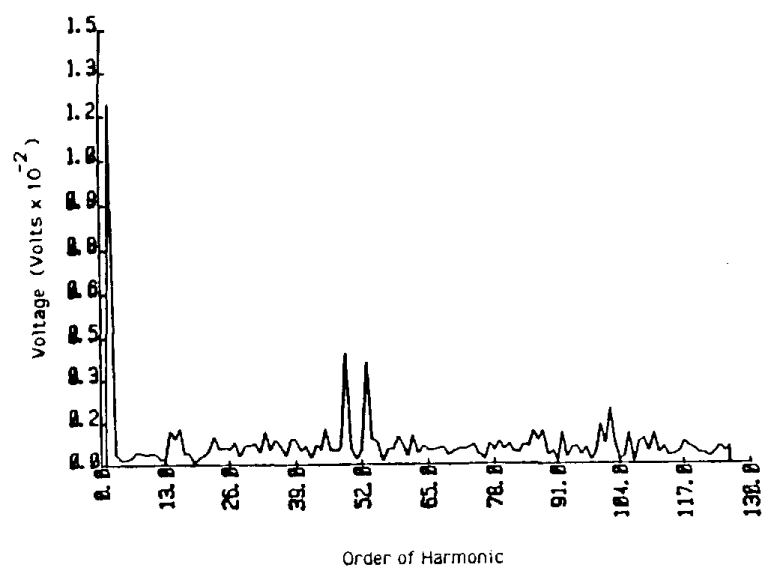

Fig. 9. Spectrum of voltage waveform in Fig. 7.

harmonic frequencies of the triangle signal, and this makes the filter design problem much simpler.

The filter used during the implementation of the test utilized the real-time sampling capability of the computer controller and employed a moving average filter of the form

$$
y(k)=\frac{1}{N}\left(1+z^{-1}+\cdots+z^{-(N-1)}\right)
$$

where $z=e^{-s T}$ and $T$ is the sampling rate $(1.0 \mathrm{~ms}$ in the prototype system). The frequency response of such a filter can be evaluated by substituting $z=e^{j \omega t}$ in (12):

$$
H(\omega)=\frac{1}{N}\left(1+e^{-j \omega T}+e^{-j 2 \omega T}+\cdots+e^{-j(N-1) \omega T}\right) .
$$

The magnitude of the transfer function as a function of $N$ is plotted in Fig. 10 for $N$ from 2 to 30 . The horizontal scale is normalized to the basic sampling frequency $1 / T$. The figure clearly shows the low-pass characteristic of the filter. The gain of the filter at zero frequency is always unity. As the value of $N$ increases, the cut-off frequency of the filter decreases. At the same time the gain of the side lobes decrease. For the actual

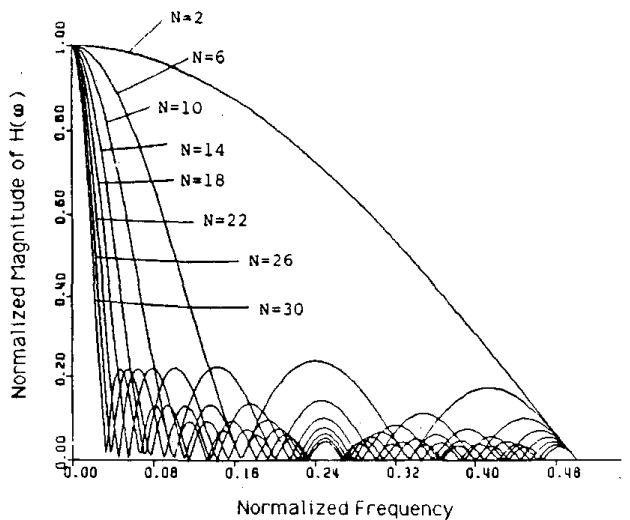

Fig. 10. Frequency response of moving average filter with increasing values of $N$.

rotor time constant test the parameter $N$ was set to 50 . It is clear that such a filter is easily implemented in a microprocessor.

\section{Test Results}

The rotor time constant of a laboratory 3-hp machine has been measured by the new measurement technique. (The nameplate data of the motor is given in the Appendix). The value of the open-circuit rotor time constant computed from information supplied by the manufacturer is $0.135 \mathrm{~s}$. From a conventional locked rotor test and a no-load test, the rotor time constant was determined to be $0.140 \mathrm{~s}$. The open-circuit rotor time constant was also measured by the automated measurement system. In this test the computer controller continually adjusts the command slip frequency until the stator voltage transient is a minimum. The entire test is carried out with a rated flux command; however, the actual flux is at its rated value only when the slip command is correct.

The filtered terminal voltages for three different cases of command slip frequencies are shown in Fig. 11. It can be noted that when the command slip frequency is twice the correct frequency, the voltage is initially too high and eventually decays the steady-state value. If the command slip frequency is one-half the correct frequency, then the voltage is initially too low. When the slip frequency is correct, the voltage transient vanishes. In all three cases the voltage error is defined as the difference between the instantaneous waveform and the steady-state value. This error is plotted against the command slip frequency in Fig. 12. The measured opencircuit rotor time constant obtained by this test was $0.120 \mathrm{~s}$. The results of Fig. 12 were obtained with a fully automated prototype test system. In Fig. 11 the values of $\omega_{s}^{*}$ were hand set to the specific values in the figure.

\section{Verification of the Test Results}

If the measured value of rotor time constant is compared to the nameplate value or to the value obtained from a lockedrotor/no-load test it would appear that an "error" of 12-16 percent was obtained. However, the locked rotor measurement is subject to its own errors. There are also inherent difficulties in obtaining identical test conditions of flux and frequency. A 

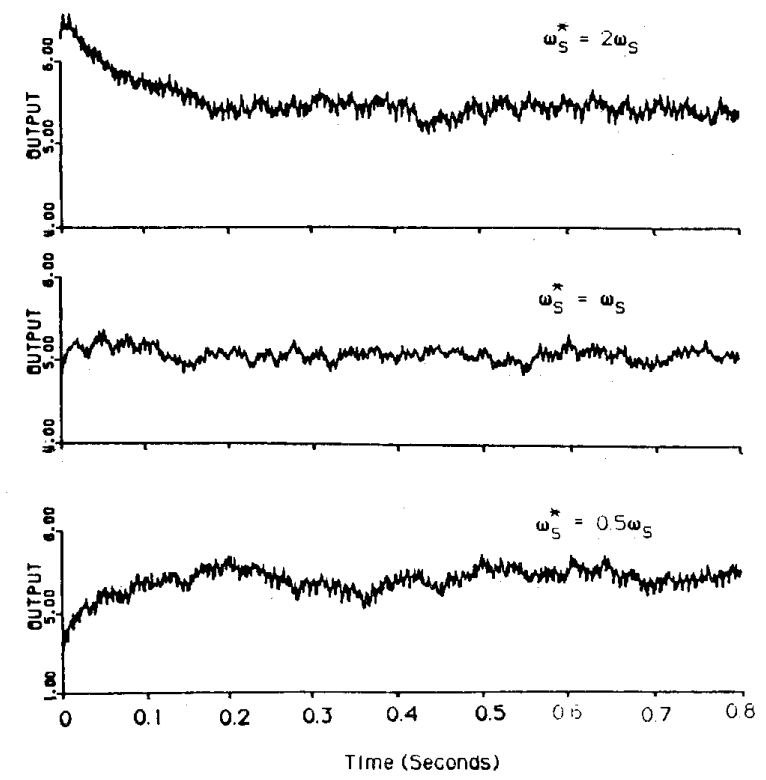

Fig. 11. Stator terminal voltage response for three different command frequencies.

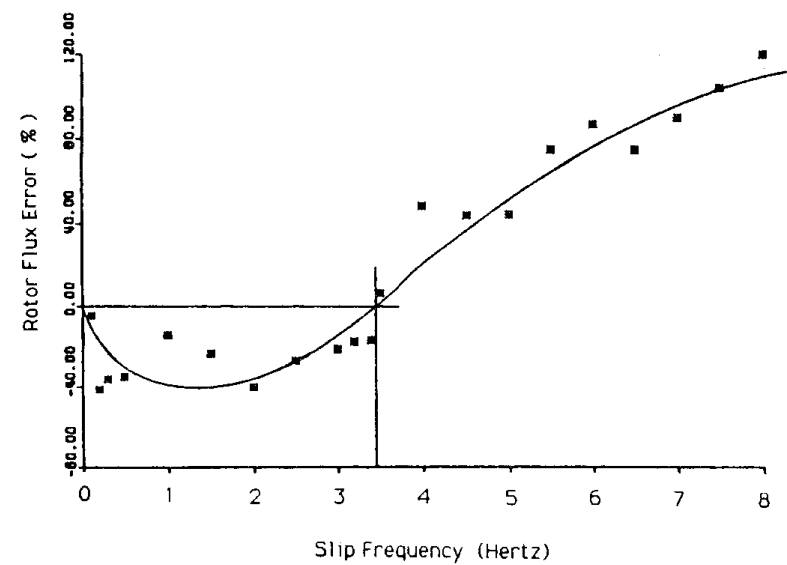

Fig. 12. Voltage error versus commanded slip frequency (3-hp machine) as measured by automated procedure.

better method of verifying the results of the time constant measurement is to directly measure the performance characteristic of the field-oriented controller resulting from the predicted time constant.

The purpose of a field-oriented drive system is to obtain a fast torque response and a linear relationship between the torque command and the output torque. While the transient torque response is difficult to measure, a check of a linear input-output torque relation can be used to verify the value of the rotor time constant. This check serves as a better method than comparing the value of the rotor time constant by another test because the performance of the drive system itself, which is the desired goal, is measured directly.

The stall torque test was carried out on a conventional dynomometer. The magnetizing current is fixed by the computer to generate a desired flux level in the motor. The torque-producing current is entered from the keyboard of the computer and then the proper slip frequency is calculated

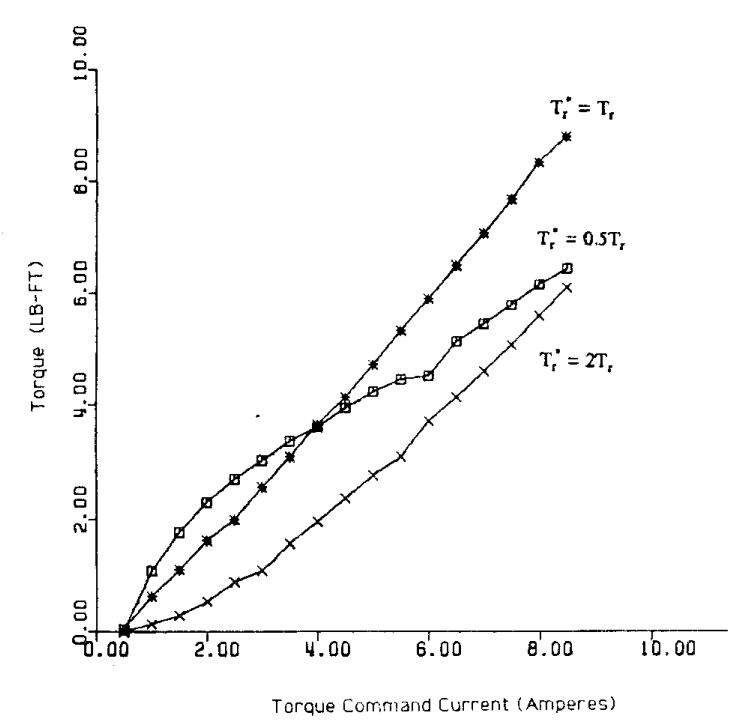

Fig. 13. Output torque versus steady-state torque command.

based on the knowledge of the rotor time constant and the magnitude of the command currents. A curve of output torque versus the input torque command current can be obtained by varying the input torque command current. The input-output torque curves for the 3-hp machine for three different gain settings are shown in Fig. 13. A linear relationship is apparent between the command and the response torque when the time constant obtained by the automated parameter measurement system is used. An expected nonlinear behavior of the response occurs when the controller time constant is set to half or twice of the value of the measured time constant. It should be mentioned that the small dead zone obtained near the value of zero command current is caused by the nonlinear behavior of the voltage-controlled oscillator of the field-oriented controller near zero frequency and is not caused by an error in the rotor time constant.

\section{Sensitivity of the Test to Machine Rating}

To be a truly automated parameter measurement system, the rotor time constant test must be able to handle a range of different motor sizes. For a reasonable range of motor size from 1 to $400 \mathrm{hp}$ the time constant typically ranges from 90 to $400 \mathrm{~ms}$. However, a high-efficiency 10 -hp motor can easily have a time constant of $400 \mathrm{~ms}$ because of the high magnetizing inductance and low rotor resistance. In this section the sensitivity of the test with respect to the value of the rotor time constant and the flux is discussed.

The rotor time constant test uses the voltage transient to detect the correctness of the time constant. The larger the voltage transient, the better will be the sensitivity of the test. There is, in effect, no upper limit to the size of time constant that can be tested for this reason. However, a lower limit does exist because of the limited sampling frequency of the computer. If the time constant becomes small, then the information contained in the voltage transient will not be sufficient to determine the rotor time constant.

Two different machine sizes are used to show the sensitivity 


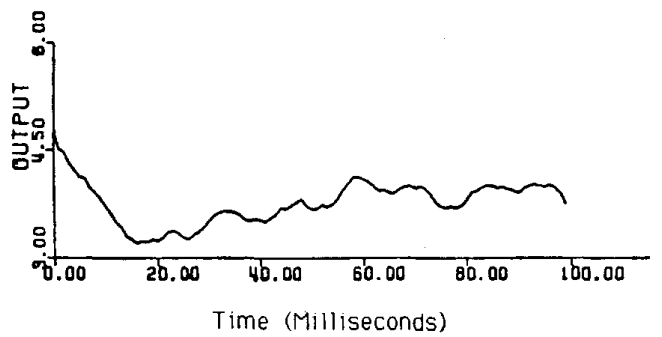

Fig. 14. Voltage transient for $1 / 3$-hp machine when estimated rotor time constant is too small $\left(\tau_{r}^{*}=0.5 \tau_{r}\right)$.

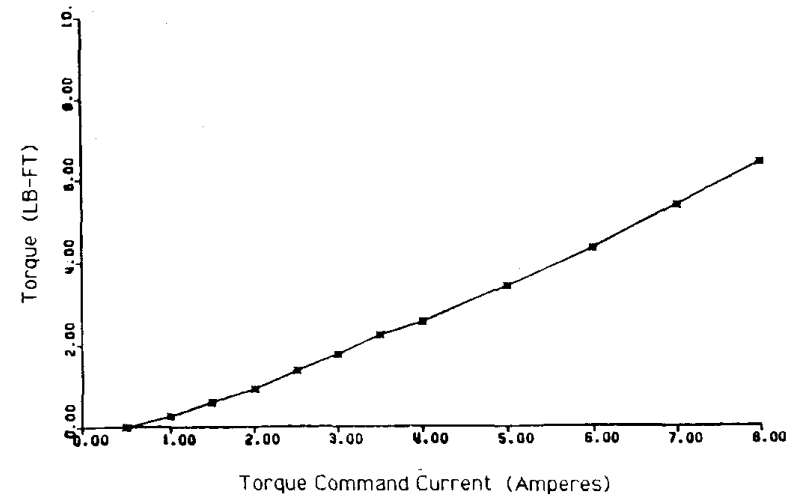

Fig. 15. Input-output torque relation for high-efficiency induction machine when controller is properly tuned by automated measurement system.

of the test with respect to the size of the rotor time constant. The first machine is a very small wound rotor machine. The rotor time constant measured by locked rotor test is $27.4 \mathrm{~ms}$. A typical test voltage transient for this motor is shown in Fig. 14. Even though the transient dies out very fast, it is still detectable. The result obtained from the automated test is 30 ms.

The second machine is a high-efficiency 7.5-hp squirrelcage motor. The motor is operated at one-third the rated flux because of the current limit of the controller. This restriction makes the rotor time constant even larger because the machine is less saturated. The rotor time constant measured by the single-phase test is $350 \mathrm{~ms}$. The result is verified by the stalled torque test, which was discussed in the last section. The output torque versus the command current is shown in Fig. 15, and the linear input-output torque relation is clearly indicated.

\section{Effect of Flux Level (Saturation)}

The voltage transient is a result of the flux transient when the controller is detuned. From the circuit of Fig. 2(b) it can be shown that the transient voltage from the instant of energization to the point of switching to a dc value can be written as

$$
\nu_{s t}=I_{s \phi} L_{m}\left(\frac{\sqrt{1+C R^{2}}}{\sqrt{1+\left(\frac{\tau_{r}}{\tau_{r}^{*}}\right)^{2} C R^{2}}} \sin \left(\omega_{e} t+\alpha\right)-1\right) e^{-t / \tau_{r}}
$$

where $C R$ is the current ratio $I_{s T}^{*} / I_{s}^{*}$.

If $C R$ is fixed, then the result indicates that the transient voltage will be directly proportional to the magnetizing

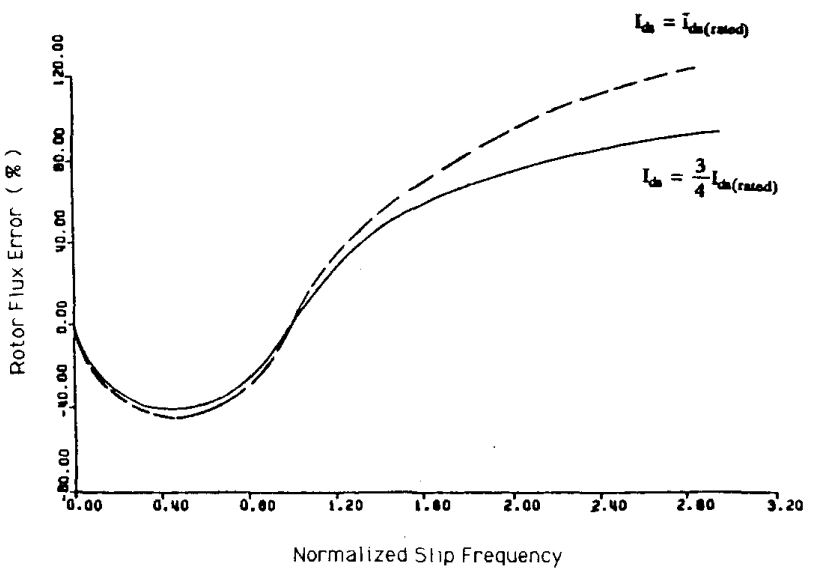

Fig. 16. Measured voltage transient versus slip frequency for different flux levels for 3-hp machine.

current. However, the expression will be modified in a real machine because of heavy saturation as demonstrated in Fig. 16. In this figure the dashed line represents rated flux operation of the 3-hp machine and the solid line represents operation at three-fourths rated flux current. Both curves are normalized to their proper tuned slip frequencies. The error clearly becomes larger as the command slip frequency increases for rated flux operation. However, the magnitude of the error is about the same when the command slip frequency is too small because of saturation.

Again the measured results can be verified by a set of the stalled torque tests. In Fig. 17 the top curve represents the motor running with rated flux and the bottom curve represents 


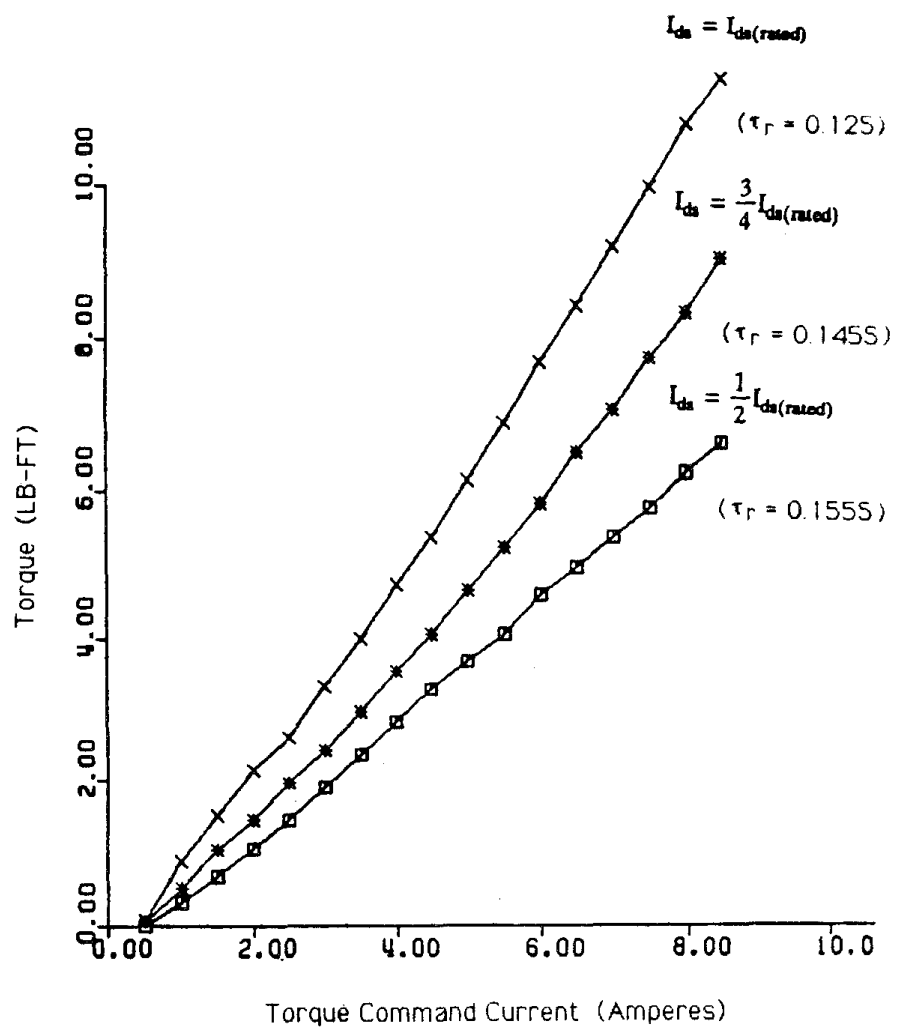

Fig. 17. Measured input-output torque curves for three different flux levels using 3-hp machine.

the case with one-half the rated flux current. Notice that the time constant varies as the flux level changes, as would be anticipated.

\section{ConClusion}

The rotor time constant of an induction motor can be measured by injecting a single-phase ac current and observing the voltage transient that occurs when this test current is switched to dc. This method is closely related to the principle of field orientation and provides results that relate directly to field-oriented operation at any selected flux level.

The implementation of a self-tuning system based on the method has been demonstrated, and the results of the selftuning process have been verified to produce the linear torque versus torque command behavior associated with field orientation. Sensitivity to test current amplitude, machine size, and saturation were examined, and the system was shown to be capable of accommodating the normal variations expected in practice.

\section{APPENDIX}

\section{Details of 3-hp Test Machine}

Nameplate data:

squirrel-cage induction machine;

3-hp, 230-V, 8.4-A;

$1725 \mathrm{r} / \mathrm{min}, 60 \mathrm{~Hz}$, three-phase, four poles.

Parameter values:

stator resistance $r_{s}=1.174 \Omega$ rotor resistance $r_{r}=0.764 \Omega$

stator leakage inductance $L_{i s}=0.00345 \mathrm{H}$

rotor leakage inductance $L_{i r}=0.00181 \mathrm{H}$

magnetizing inductance $L_{m}=0.0761 \mathrm{H}$

iron loss resistance $R_{m}=818.4 \Omega$.

\section{REFERENCES}

[1] K. Nordin, D. W. Novotny, D. S. Zinger. "Influence of motor parameter deviations in feedforward field oriented systems," IEEE Trans. Ind. Appl., vol. IA-21, pp. 1009-1015. July/Aug. 1985.

[2] M. Koyama et al., "Effects of parameter change on coordinate control system of induction motor," in Conf. Record IPEC (Japan) Conf., Tokyo, Japan, Mar. 1983, pp. 684-695.

[3] L. J. Garces, "Parameter adaption for the speed-controlled static ac drive with a squirrel-cage induction motor," IEEE Trans. Ind. Appl., vol. IA-16, pp. 173-178, Mar./Apr. 1980.

[4] T. Matsuo and T. A. Lipo, "Rotor resistance identification in the field oriented control of a squirrel cage induction motor." IEEE Trans. Ind. Appl, vol. IA-2I, pp. 624-632, May/June 1985.

[5] R. Krishnan and F. C. Doran, "A method of sensing line voltages for parameter adaptation of inverter-fed induction motor servo drives. " in Conf. Record IEEE-IAS Ann. Meeting, Oct. 6-11, 1985. pp. 570577.

[6] F. Loser and Ph. K. Sattler, "Identification and compensation of the rotor temperature of ac drives by an observer," in Conf. Record IEEE-IAS 1984 Annual Meeting, Chicago, IL. pp. 532-537.

[7] K. Hasse, "Zur Dynamik Drehzahlgeregelter Antriebe Mit Stromrichtergespeisten Asynchron-Kurzschlublaufermaschinen," Ph.D. Dissertation, Tech. Hoch. Darmstadt, July 17, 1969.

[8] F. Blaschke, "A new method for the structural decoupling of ac induction machine," in Conf. Record IFAC Symp.. Dusseldorf. Germany, Oct. 1971, pp. 1-15 (6.3.1).

[9] W. Leonhard, Control of Electrical Drives. New York: SpringerVerlag, 1985.

[10] D. W. Novotny and T. A. Lipo, "Principles of vector control and field orientation," in Short Course Notes "Introduction to field orientation 
and high performance ac drives," of IEEE-IAS Ann. Meeting, Oct 6-7 1985, pp. 2-1 to $2-65$.

[11] T. M. Rowan and R. J. Kerkman, "A new synchronous current regulator and an analysis of current regulated PWM inverters," in Conf. Rec. IEEE-IAS Ann. Meeting, Oct. 6-11, 1986, pp. 487-495.

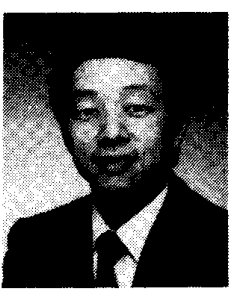

Chu-Gen Wang was born in Shanghai, China, in 1959. He received the B.S.E.E., M.S.E.E., and $\mathrm{Ph} . \mathrm{D}$. degrees in electrical engineering from the University of Wisconsin-Madison in 1985 .

$\mathrm{He}$ is currently working for the Motion Control Operations Department of Gould Inc.

Dr. Wang is a member of Sigma $\mathrm{Xi}$

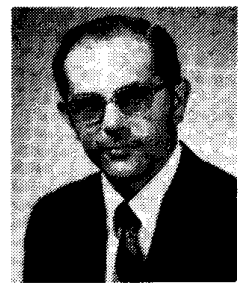

Donald W. Novotny (M'62-SM'77-F'87) received the B.S. and M.S. degrees in electrical engineering from the Illinois Institute of Technology, Chicago, in 1956 and 1957, respectively, and the Ph.D. degree from the University of Wisconsin-Madison in 1961.

Since 1961 he has been a member of the faculty at the University of Wisconsin-Madison, where he is currently Professor and Co-Director of the Wisconsin Electric Machines and Power Electronics Consortium (WEMPEC). He served as Chairman of the Electrical and Computer Engineering Department from 1976 to 1980 and as an Associate Director of the University-Industry Research Program from 1972 to 1974 and from 1980 to the present. He has been active as a Consultant to many organizations and a Visiting Professor at Montana State University,
Boseman, the Technical University of Eindhoven, Eindhoven, The Netherlands, the Catholic University of Leuven, Leuven, Belgium, and a Fulbright Lecturer at the University of Ghent, Ghent, Belgium. His teaching and research interests include electric machines, variable-frequency drive systems, and power electronic control of industrial systems.

Dr. Novotny is a member of the IEEE, the ASEE, Sigma Xi, Eta Kappa $\mathrm{Nu}$, and Tau Beta Pi, and is a Registered Professional Engineer in the State of Wisconsin.

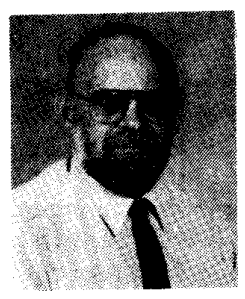

Thomas A. Lipo (M'64-SM'71-F'87) received the B.E.E. and M.S.E.E. degrees from Marquette University, Milwaukee, WI, in 1962 and 1964 respectively, and the $\mathrm{Ph} . \mathrm{D}$. degree in electrical engineering from the University of Wisconsin in 1968. He was an NRC Postdoctoral Fellow at the University of Manchester Institute of Science and Technology, Manchester, England, during 19681969.

From 1969 to 1979 he was an Electrical Engineer in the Power Electronics Laboratory of Corporate Research and Development of the General Electric Company, Schenectady, NY. He is currently a Professor in the Department of Electrical and Computer Engineering, University of Wisconsin-Madison.

Dr. Lipo is Past Chairman of the IAS Industrial Drives Committee and PES Induction Machines Subcommittee. He also serves on the IAS Electric Machines Committee and on the PES Synchronous Machine and Electric Machine Theory Subcommittees. He is a member of the Steering Committee for the International Conference on Electrical Machines, an Associate Editor of the journal Electric Machines and Power Systems, and Editor of the IEEE TRANSACTIONS ON POWER ELECTRONICS. In 1986 he was the recipient of the IAS Outstanding Achievement Award for his contributions to the motor drive industry. He is a member of Pi Mu Epsilon, Eta Kappa Nu, Tau Beta Pi, and Sigma Xi. 